\title{
Studies on the Nephrotoxicity of Aminoglycoside Antibiotics and Protection from These Effects (3) Protective Effect of Latamoxef against Tobramycin Nephrotoxicity and Its Protective Mechanism
}

\author{
Ryoji KOJIMA, Mikio ITO and Yoshio SUZUKI \\ Department of Pharmacology. Faculty of Pharmacy, Meijo University. \\ 15 Yagoto Urayama, Tenpaku-ku, Nagoya 468, Japan
}

Accepted August 4, 1986

\begin{abstract}
Effect of latamoxef (LMOX) against tobramycin (TOB)-induced nephrotoxicity was studied in rats. Treatment with TOB $(90 \mathrm{mg} / \mathrm{kg} / \mathrm{day}$, s.c.) alone resulted in marked increases in the activities of urinary enzymes such as lactate dehydrogenase, $\mathrm{N}$-acetyl- $\beta$-D-glucosaminidase and lysozyme, urinary protein content and blood urea nitrogen, which peaked on the 7 th or 10th day. The combination with LMOX (500, 1000 or $2000 \mathrm{mg} / \mathrm{kg} / \mathrm{day}$, s.c.) significantly suppressed increases in the parameters with TOB alone. The extent of this suppression roughly depended on the LMOX dosage. Although TOB alone caused pronounced histological changes such as extensive cortical proximal tubular cell necrosis, residual tubular basement membrane and cast formations in the renal cortex and medulla on the 7 th day, these changes were apparently suppressed by combination with LMOX. In addition, intrarenal TOB concentrations in the rat given TOB alone were about 350, 500 and $1000 \mu \mathrm{g} / \mathrm{g}$ tissue wet weight at $3 \mathrm{hr}$. on day 3 and on day 5 , respectively. On the other hand, there was a significant reduction $(30-60 \%)$ in intrarenal TOB concentration by combination with LMOX. These results indicate that combination with LMOX obviously protects the rat kidney from TOB nephrotoxicity, and the protective effect may be partially due to suppression of intrarenal accumulation of TOB by LMOX.
\end{abstract}

Aminoglycoside antibiotics (AGs), which are agents against infectious diseases, have severe side effects such as nephrotoxicity (1), ototoxicity (2) and neuromuscular junction blocking (3). Because of these side effects, the use of AGs has been limited in clinical situations. Recently, AGs have been widely used in combination with other antibiotics such as $\beta$-lactam antibiotics, which have nephrotoxicity as a side effect. Thus, it is likely that the adverse effects of AGs are augmented by these drug combinations.

Previous study in this laboratory, however, has demonstrated that latamoxef (LMOX), an oxacephem antibiotic, can protect against gentamicin (GM)-induced nephrotoxicity in rats rather than augment it (4). The mecha- nisms by which LMOX protects the kidneys from GM nephrotoxicity remain unclear. Many investigators have reported that AGs are probably transported from the renal luminal side to the renal tubular cells due to pinocytosis: they accumulate in the kidney, especially in the renal cortex in animals (5. 6 ) and humans (7). There is a close relationship between the accumulation of $A G s$ in the kidney and the extent of the nephrotoxicity (8). In the present study, the combination with LMOX protected against the rat nephrotoxicity induced by tobramycin (TOB), an aminoglycoside antibiotic, which resembles GM in molecular structure and bacteriocidal activity. The protective mechanism of LMOX was also investigated by measuring the intrarenal and blood TOB 
concentrations in rats.

\section{Materials and Methods}

Animals: Male Sprague-Dawley rats weighing approx. $230 \mathrm{~g}$ (Shizuoka Laboratory Animal Center) were used. These animals were housed in an air-conditioned room at $23 \pm 1^{\circ} \mathrm{C}$.

Drugs: Drugs used were tobramycin (TOB, Shionogi Co.. Ltd.) and latamoxef sodium (LMOX, Shionogi Co., Ltd.). Both TOB and LMOX were dissolved in saline.

Drug treatment: The rats were given daily s.c. doses of TOB $(90 \mathrm{mg} / \mathrm{kg})$ alone or $T O B$ and LMOX (500, 1000 or $2000 \mathrm{mg} / \mathrm{kg}$ ) for 15 consecutive days. These two antibiotics were simultaneously given at separate sites.

Urine and blood collections: The rats were given $8 \mathrm{ml}$ of water and housed individually in stainless steel metabolic cages after weighing and receiving the s.c. injections.

Twenty-four hour urine was collected, and blood was obtained from the tail vein on the 1st. 3rd, 5th, 7th, 10th and 15th days. The urine was centrifuged at $3,000 \mathrm{rpm}$ for $15 \mathrm{~min}$, and the resultant supernatant was used for measuring the protein content and enzyme activities. Serum separated from blood was used to determine urea nitrogen content.

Measurements of biochemical parameters in urine and blood: Urinary protein content was measured in accordance with the sulfosalicylic acid method (9) and calculated as $\mathrm{mg}$ per $24 \mathrm{hr}$ urine. Urinary $\mathrm{N}$-acetyl- $\beta$ D-glucosaminidase (NAG, EC 3.2.1.30) activity was assayed by the method of Hasebe (10) using p-nitrophenyl-N-acetyl- $\beta$-Dglucosaminide (Sigma) as a substrate. The enzyme activity was expressed as $\mathrm{mU} / 24 \mathrm{hr}$ urine. Urinary lactate dehydrogenase ( $\mathrm{LDH}$, EC 1.1.1.27) activity was measured using a LDH Linia-Neo 3A kit (Shinotest), and the activity was represented in Wróblewski units (U) per $24 \mathrm{hr}$ urine. For measuring urinary lysozyme (LZM, EC 3.2.1.17) activity, micrococcus Iysodeikuticus was used as a substrate. The enzyme activity was measured by the method of Hasebe (10) and expressed as $\mu \mathrm{g} / 24 \mathrm{hr}$ urine. Blood urea nitrogen (BUN) was analyzed by the method reported by Searcy and Cox (11) and expressed as mg/dl serum.
Histological studies: The rats from each group were anesthetized with i.p. injection of sodium pentobarbital $(32.4 \mathrm{mg} / \mathrm{kg}$ ) on the 5th and 7th days after beginning drug treatment. The kidneys were quickly removed, divided into two, fixed in neutral buffered formalin and embedded in paraffin. The paraffin sections were stained with Periodic Acid Schiff (PAS) for light microscopy.

Measurements of intrarenal and blood TOB concentrations: In order to examine the effect of LMOX on intrarenal and blood TOB concentrations, rats were given TOB $(90$ $\mathrm{mg} / \mathrm{kg}$, s.c.) alone or TOB in combination with LMOX (2000 mg/kg. S.c.) daily for 7 consecutive days. At 3,6, 9 and $24 \mathrm{hr}$ and on the 3 rd. 5 th and 7 th days after initiating drug treatment, the animals were anesthetized with sodium pentobarbital $(32.4 \mathrm{mg} / \mathrm{kg}$ ), and a midline abdominal incision was made to expose the abdominal aorta and left kidney. Perfusion of the left kidney with $0.1 \mathrm{M}$ saline-phosphate buffer $(\mathrm{pH}$ 8.1) was undertaken to wash out the blood. Then, the kidney was rapidly removed. After weighing. the kidneys were minced and diluted $1: 7$ with a $0.1 \mathrm{M}$ saline-phosphate buffer $(\mathrm{pH} 8.1)$. followed by homogenation with a teflon glass homogenizer for $1 \mathrm{~min}$. The homogenate was centrifuged at $4,000 \mathrm{~g}$ for $10 \mathrm{~min}$. The resultant supernatant was used for measuring TOB concentration by enzyme immunoassay (EMIT, Syva Corp.) in accordance with the method of Saionji et al. (12). In addition. blood samples were obtained from the jugular vein at 3,6 and $9 \mathrm{hr}$ after beginning drug treatment. Then, the TOB concentration in the separated serum was measured. Intrarenal and serum TOB concentrations were expressed as $\mu \mathrm{g} / \mathrm{g}$ wet tissue weight and $\mu \mathrm{g} / \mathrm{ml}$ serum, respectively.

Statistical analysis: The results in the text and figures are expressed as the means \pm S.E.M. Student's t-test was used for statistical analysis. The percent suppression in the results was derived from the following formula: $\frac{T-L}{T-N} \times 100$ or $\frac{T-L}{T} \times 100$ (where $T=T O B, L=T O B$ and $L M O X$, and $N=$ Normal)

\section{Results}

\section{Effects of TOB alone and in combination}


with LMOX on biochemical parameters in urine and blood

Urinary protein content: When TOB alone was given to rats, their urinary protein contents began to elevate from the 5th day and peaked on the 7 th day. Thereafter, urinary protein content decreased in spite of the continuous administration of TOB and returned to nearly the normal level by the 15 th day. However, concurrent treatment with TOB and LMOX significantly suppressed the increase in their urinary protein content with TOB alone by 70 to $90 \%$. The suppression was the greatest when the LMOX $(2000 \mathrm{mg} / \mathrm{kg}$. s.c.) was administered simultaneously with TOB (Fig. 1).

Urinary LDH activity: As shown in Fig. 1, the urinary LDH activity was significantly increased by administration of TOB alone. This elevation began from the 3rd day and reached maximum on the 7 th day. On the other hand, the increase in activity induced by TOB alone was suppressed by 30 to $90 \%$ by combination of TOB with LMOX. This suppression by LMOX roughly depended on the LMOX dosage.

Urinary NAG activity: The urinary activity of NAG, one of the Iysosomal enzymes of renal tubular cells in rats receiving TOB

\section{protein}

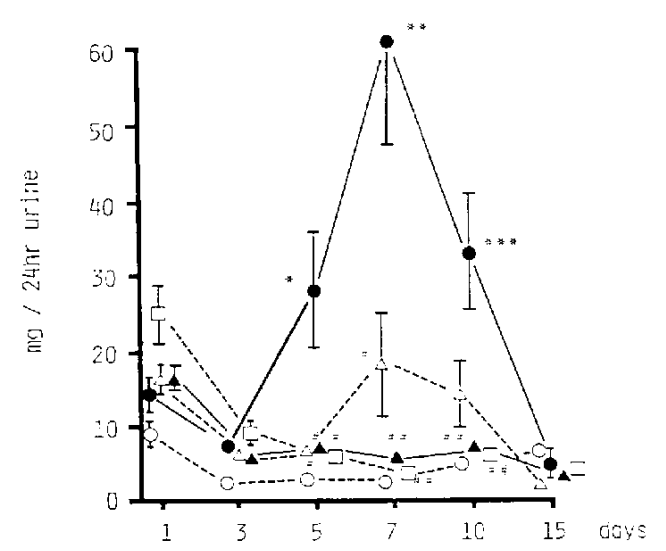

alone, increased to approx. 20 -fold that of normal rats by the 7th day.

The concurrent administration of TOB and LMOX showed 40 to $90 \%$ suppression of urinary NAG activity as compared with that of TOB alone (Fig. 2).

Urinary LZM activity: The urinary LZM activity significantly increased by TOB alone. whereas these increases were suppressed about 60 to $100 \%$ by combination with LMOX. This parameter in the rats given TOB and LMOX (2000 mg/kg. s.c.) was maintained at almost normal levels throughout the experimental period (Fig. 2).

BUN: As shown in Fig. 3, treatment with TOB alone showed an increase in BUN which peaked on the 10th day. On the other hand, combination with LMOX (2000 mg/kg, s.c.) significantly suppressed the BUN elevation produced by TOB by about $50 \%$ on the 10 th day.

2. Effects of TOB alone and in combination with LMOX on histological findings of kidneys

Photograph 1 presents light micrographs of kidney sections from rats given TOB $(90 \mathrm{mg} /$ $\mathrm{kg} /$ day, s.c.) or TOB and LMOX $(2000 \mathrm{mg} /$ $\mathrm{kg} /$ day, s.c.) on day 5 . When TOB alone was given, the most conspicuous finding was the

Fig. 1. Effects of tobramycin (TOB) alone and in combination with latamoxef (LMOX) on urinary protein content and lactate dehydrogenase activity. (O): normal, (O): TOB, $90 \mathrm{mg} / \mathrm{kg}$. ( $\triangle$ ): TOB.

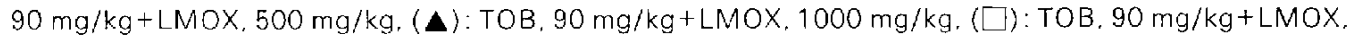
$2000 \mathrm{mg} / \mathrm{kg}$. Each plot represents the mean \pm S.E. of 7 rats. ${ }^{*}: P<0.05,{ }^{* *}: P<0.01,{ }^{* * *}: P<0.001$, compared to normal. $P<0.05$. P<0.01, $P<0.001$. compared to TOB alone. 

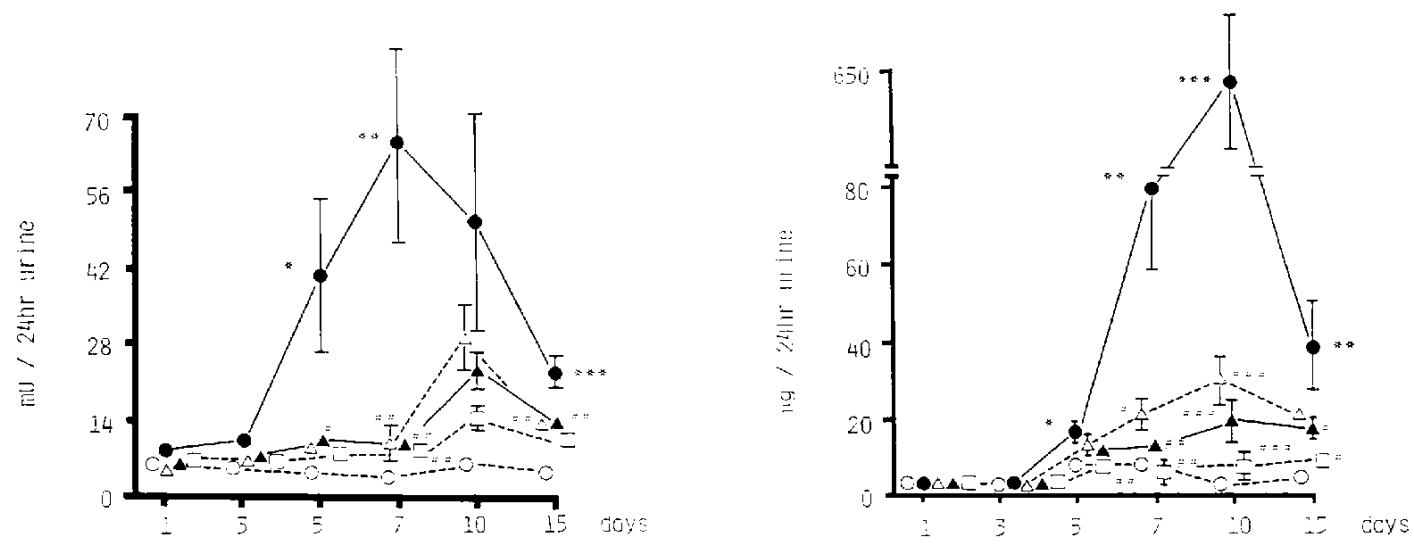

Fig. 2. Effects of tobramycin (TOB) alone and in combination with latamoxef (LMOX) on urinary $N$-acetyl- $\beta$-D-glucosaminidase and lysozyme activities. ${ }^{*}: P<0.05,{ }^{* *}: P<0.01,{ }^{* *}: P<0.001$, compared to normal. $: P<0.05$. $P<0.01$. $P<0.001$, compared to TOB alone. Other explanations are as in Fig. 1.

blood urea nitrogen

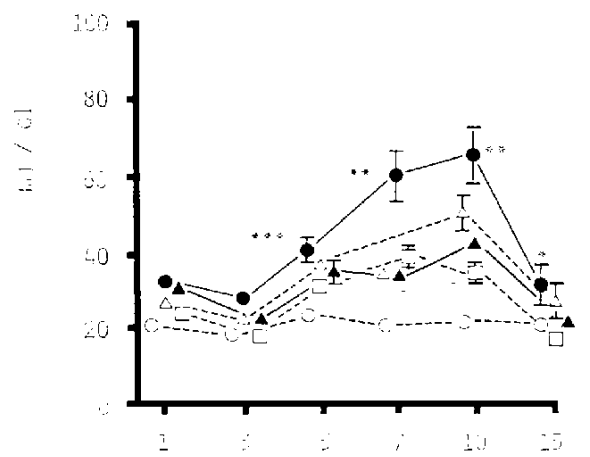

Fig. 3. Effects of tobramycin (TOB) alone and in combination with latamoxef ( $L M O X$ ) on blood urea nitrogen content. * P<0.05, **:P<0.01. *** $P<0.001$. compared to normal. *: $P<0.05$, * $P<0.01$ compared to TOB alone. Other explanations are as in Fig. 1.

appearance of a large number of PASpositive droplets within the proximal tubular cells. There were few necrotic cells at this time (Photo 1a). However, combination with LMOX (2000 $\mathrm{mg} / \mathrm{kg}$, s.c.) revealed almost normal proximal tubular cells with very few PAS-positive droplets (Photo 1b).
On day 7 , treatment with TOB alone showed extensive proximal tubular cell necrosis and residual tubular basement membrane in the renal cortex. In addition, there were numerous casts packed within the proximal and distal tubular lumen in the renal cortex and medulla and partial distal tubular injury in the cortex. The glomeruli did not show any changes under light microscopy. No evidence of regenerating tubular epithelial cells was observed by this time (Photo 2a). Combination with LMOX (500 or $1000 \mathrm{mg} / \mathrm{kg}$. S.C.) revealed some necrotic cells. while the concurrent administration of TOB and LMOX (2000 mg/kg, s.c.) showed almost normal proximal tubular cells in the renal cortex. indicating that the TOB-induced renal tubular injury was prevented by the combination with LMOX (Photo 2b). In addition. on day 15 , renal histological findings of the rats given $\mathrm{TOB}$ alone or the combination with LMOX were similar to those of GM alone or in combination with LMOX as previously described by the present investigators (4).

\section{Effects of LMOX on intrarenal and blood} TOB concentrations

Intrarenal TOB concentration: The intrarenal $T O B$ concentration in rats receiving $\mathrm{TOB}(90 \mathrm{mg} / \mathrm{kg}$ ) alone was $363.6 \pm 15.0$ 

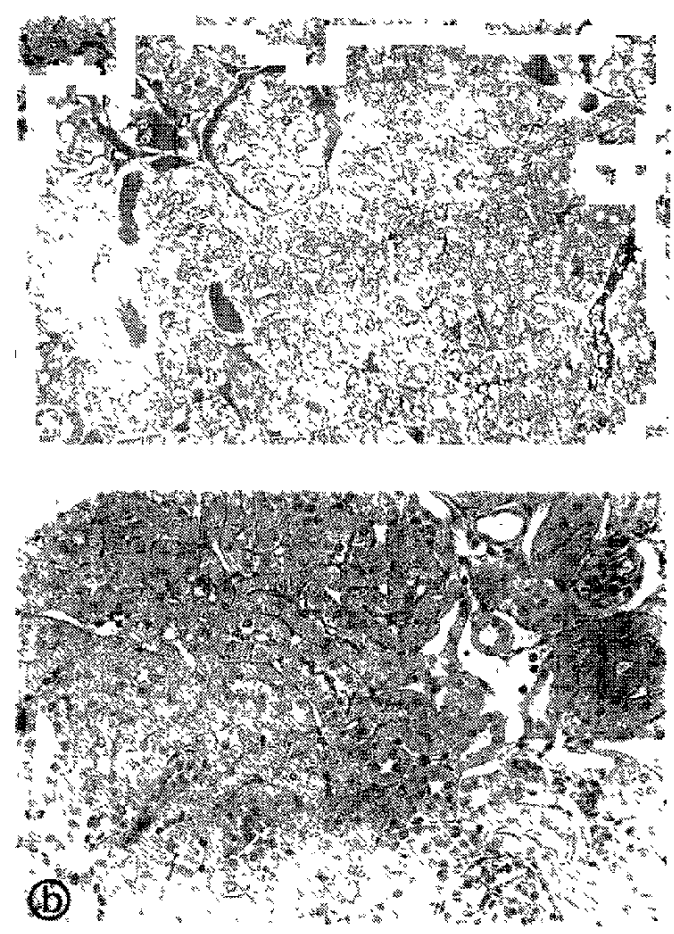

Photo 1 Light micrograph of rat kidney on the 5 th day (PAS stan) a TOB alone--note a large number of PAS-positive droplets in the proximal tubular cells (arrow head), and these cells are about to be destroyed b TOB +LMOX. $200 \mathrm{mg} / \mathrm{kg}$-most of tubules show normal characteristics $\times 200$

$\mathrm{kg} / \mathrm{g}$ tissue wet weight at $3 \mathrm{hr}$ after s c injection Then, it reached a maximum value of $10368 \pm 573 \mathrm{\mu g} / \mathrm{g}$ tissue wet weight on day 5 and declined sharply on day 7 On the other hand, the concurrent administration of TOB and LMOX (2000 $\mathrm{mg} / \mathrm{kg})$ suporessed the intrarenal TOB accumulation by $50 \%$ at $3 \mathrm{hr}(1530 \pm 130 \mu \mathrm{g} / \mathrm{g}$ tissue wet weight), and this suppression contınued from 3 to $24 \mathrm{hr}$ Thereafter, there were 30 and 50\% reductions of intrarenal TOB concentrations on days 3 and 5 , respectively, in rats recelving TOB and LMOX $(2000 \mathrm{mg} / \mathrm{kg})$ However, no significant difference was observed between both groups on day 7

Serum TOB concentration: Serum TOB concentrations of rats that recelved TOB alone and In combination with LMOX (2000 $\mathrm{mg} / \mathrm{kg}$ ) were $339 \pm 33 \mu \mathrm{g} / \mathrm{ml}$ and $301 \pm 35$ $\mathrm{kg} / \mathrm{ml}$ at $3 \mathrm{hr}$ after Sc injection, respectively, and there was no significant difference
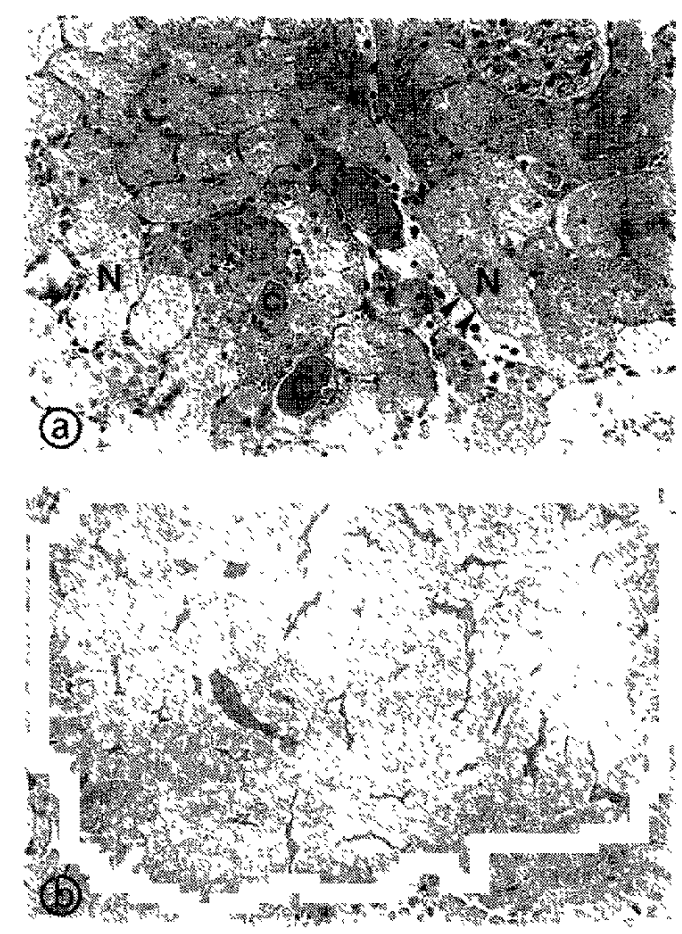

Photo 2 Light micrograph of rat kidney on the 7 th day (PAS staın) a TOB alone-note the extended necrosis of proximal tubular epithelıal cells (N), casts formation (C) and residual tubular basement membrane (arrow head) b TOB + LMOX. $200 \mathrm{mg} /$ $\mathrm{kg}$-almost normal cells are observed. $\times 200$

between them throughout the experimental period

\section{Discussion}

Urinary LDH, NAG and LZM activities were measured as marker enzymes of TOB-Induced nephrotoxicity It has already been reported by several investigators that there are increases in urinary activities of these enzymes when nephrotoxic agents are administered to rats $(13,14)$ Our previous study has demonstrated that the treatment of rats with GM or $\mathrm{HgCl}_{2}$ induced significantly increased urinary enzyme actıvities, leading to the conclusion that these activities are suitable markers for detectıng nephrotoxicity by nephrotoxic agents (15). In the present study, TOB, one of the AGs, was used as a nephrotoxic agent, and the protective effect of LMOX against TOB-Induced nephrotoxicity was investigated in rats by 
renal histological studies and measurement of biochemical parameters including urinary LDH, NAG and LZM activities.

The urinary enzyme activities, protein content and BUN significantly increased by treatment with TOB alone, whereas increases in these parameters were significantly suppressed by combination with LMOX (Figs. $1-3)$. In addition, histological study revealed extensive cell necrosis in the renal cortex of rats receiving TOB alone (Photo 2a). However, there was no light-microscopic evidence of these histological changes in rats receiving TOB and LMOX (Photo $2 \mathrm{~b}$ ). These results indicate that coadministration of LMOX protects kidneys from TOBinduced nephrotoxicity, and this result was similar to our previous results with GM (4). In order to clarify the protective mechanisms of LMOX, the effects of LMOX on intrarenal and blood TOB concentrations were studied in rats. When TOB alone was given to rats, the intrarenal TOB concentration maintained almost the same levels from 3 to $24 \mathrm{hr}$. Likewise, the intrarenal TOB concentration in combination with LMOX also showed consistently lower levels than that of TOB alone until $24 \mathrm{hr}$ (Fig. 4).

These evidences suggest that the intrarenal TOB accumulation was almost saturated by $3 \mathrm{hr}$, and the suppressive effect of LMOX on intrarenal TOB accumulation appears by $3 \mathrm{hr}$ and continues until $24 \mathrm{hr}$. The intrarenal TOB concentration peaked on the 5 th day and then declined rapidly on the 7th day (Fig. 4). This reduction appears to be due to the severe necrosis of cells accumulating TOB as shown in Photo 2a. The combination with LMOX suppressed the accumulation of TOB in the kidneys throughout the experimental period except for the 7th day. Therefore, it is suggested that the protective mechanism of LMOX against TOB nephrotoxicity may be attributed to the suppression of intrarenal TOB accumulation by LMOX.

Dellinger et al. (16, 17) have reported that the treatment with GM $(12 \mathrm{mg} / \mathrm{kg} /$ day. s.c.) and cephalothin (CET, $400 \mathrm{mg} / \mathrm{kg} / \mathrm{day}$, s.c.) reduces the renal tubular cell necrosis induced by GM and that the protective mechanism of CET may be due to the suppression of accumulation of GM in the kidneys. The present finding was in agreement with that of Dellinger et al. (17) in terms of the suppression of intrarenal aminoglycoside antibiotic concentration. Although it was not clear whether LMOX affected the intrarenal GM accumulation in this study, it is likely that the protective mechanism of LMOX against GM-induced nephrotoxicity may also be similar to the results of the present study because of the similarity in GM and TOB molecular structure.

Just and Habermann (18), and Silverblatt and Kuehn (19) have reported that GM binds to rat renal brush border membrane (BBM) and is sequestered within lysosomes by pinocytosis. Sastrasinh et al. (20) con-
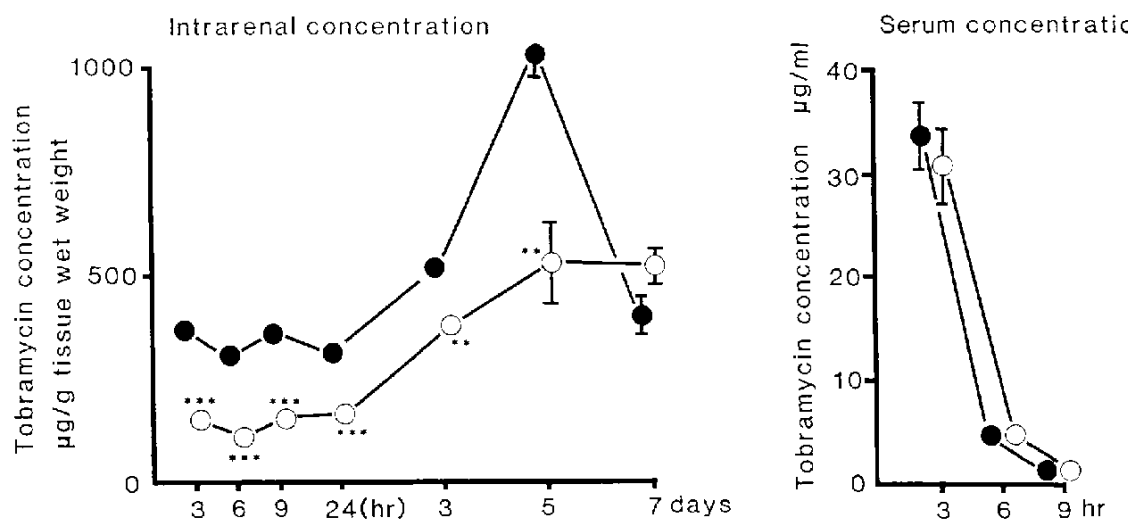

Fig. 4. Effect of latamoxef (LMOX) on intrarenal and blood concentrations of tobramycine (TOB). (O): TOB, $90 \mathrm{mg} / \mathrm{kg}:$ (O): TOB, $90 \mathrm{mg} / \mathrm{kg}+\mathrm{LMOX}, 2000 \mathrm{mg} / \mathrm{kg} . \quad$ **: P<0.01, **: P<0.001, compared to TOB alone. Each plot represents the mean $\pm S . E$. of 7 rats. 
cluded that acidic phospholipids, including phosphatidylinositol, were the renal BBM binding sites for $A G$ and that the binding was due to a charge interaction between the acidic phospholipids of BBM and the polybasic AGs. Luft and Kleit (21) indicated that the intrarenal TOB concentration after a single s.c. injection in rats gradually declined with excretion of the drug into the urine over 11 days, which suggests that the tubular cells have an elimination pathway of TOB into the lumen. Therefore, in regard to the suppressive mechanism of the intrarenal TOB accumulation by LMOX, it is conceivable that LMOX may inhibit TOB binding to renal BBM or pinocytosis which finally transports TOB to the lysosomes: LMOX, moreover, may accelerate the elimination of TOB from tubular cells into the urine. Bennett et al. (22) have reported that high sodium intake reduces the renal cortical concentration of $G M$ in rats. Therefore, since LMOX was injected as a sodium salt in the present study, sodium intake with s.c. injection of LMOX also may be partially associated with the reduction of intrarenal TOB concentration by LMOX. Additionally, since LMOX is filtrated through the glomerulus mainly without a transport from the renal antiluminal side to the cytosol in rats (23). LMOX may have an effect on intrarenal TOB accumulation in the luminal side.

In this study, when LMOX was given to rats simultaneously with TOB, LMOX did not show any effect on the serum TOB concentration (Fig. 4). Therefore, the protective effect of LMOX cannot be explained by the serum TOB levels.

Recently, it has been considered in the pathogenesis of AGs nephrotoxicity that the tubular lysosomes accumulating AGs molecules or myeloid bodies may be disrupted and then, numerous lysosomal enzymes may be released into cytosol, leading to cell necrosis (24), because AGs produce myeloid bodies within lysosomes with phospholipidosis (25). Ngaha (26) and Fry and Plummer (27) reported that CER, a $\beta$-lactam antibiotic, exerted a stabilizing effect on the rat kidney lysosomal membranes in vivo and in vitro. This, therefore, raises another possibility for the LMOX protective mecha- nism: LMOX, which has a $\beta$-lactam structure in its molecular structure, also may stabilize Iysosomal membranes, and LMOX may prevent the renal tubular injury induced by TOB.

In conclusion, the present results indicate that combination of TOB with LMOX obviously protects the kidneys from TOB nephrotoxicity, and this effect may be partially related to the suppression by $L M O X$ of TOB renal accumulation.

Acknowlegement: We are grateful to Shionogi $\mathrm{Co}$.. Ltd., for supplying TOB and LMOX.

\section{References}

1 Bennett, W.M.: Aminoglycoside nephrotoxicity. Nephron 35, 73-77 (1983)

2 Lodhi, S., Weiner, N.D., Mechigian, I. and Schacht, J: Ototoxicity of aminoglycosides correlated with their action on monomolecular films of polyphospho-inositides. Biochem. Pharmacol. 29, 597-601 (1980)

3 Farley, J.M., Wu, C.H. and Narahashi, T.: Mechanism of neuromuscular block by streptomycin: A voltage clamp analysis. J. Pharmacol. Exp. Ther. 222, 488-493 (1982)

4 Kojima, R. and Suzuki, Y.: Studies on the nephrotoxicity of aminoglycoside antibiotics and protection from these effects (2). Protective effect of latamoxef against gentamicin nephrotoxicity. Folia Pharmacol. Japon. 84, 463-469 (1984) (Abs. in English)

5 Just, M., Erdmann, G. and Habermann, E.: The renal handling of polybasic drugs. 1. Gentamicin and aprotinin in intact animals. Naunyn Schmiedebergs Arch. Pharmacol. 300, 57-66 (1977)

6 Pastoriza-Munoz, E., Bowman, R.L. and Kaloyanides, G.J.: Renal tubular transport of gentamicin in the rat. Kidney Int. 16, 440-450 (1979)

7 De Broe, M.E., Daulus, G.J., Verpooten, G.A., Roels, F., Buyssens, N., Wedden, R., Van Hoof, F. and Tulkens, P.M.: Early effects of gentamicin. tobramycin, and amikacin on the human kidney. Kidney Int. 25, 643-652 (1984)

8 Schentag, J.J., Jusko, W.J., Vance, J.W., Cumbo, T.J., Abrutyn, E. and Plaut, M.E.: Aminoglycoside nephrotoxicity in relation to tissue accumulation. In Nephrotoxicity. Edited by Fillastre, J.P., p. 63-81, Masson Publishing U.S.A. Inc., New York (1978)

9 Bradley, G.M. and Benson, E.S.: Examination of the urine. In Todd-Sanford Clinical Diagnosis by 
Laboratory Methods (15th ed), Edited by Davidson, I. and Henry, J.B., p. 74-75, W.B. Saunders Company. Philadelphia (1974)

10 Hasebe, K: Biochemical studies on synovial fluid. Fukushima J. Med. Sci. 15, 35-44 (1968)

11 Searcy, R.L. and Cox, F.M.: A modified technique for ultramicro estimation of urea nitrogen. Clin. Chim. Acta 8, 810-812 (1963)

12 Saionji, K., lizuka, K. and Sakano, Y.: A comparison of four methods for determination of tobramycin in serum on the basis of bioassay: High-performance liquid chromatography, EMIT, substrate-labelled fluorescent immunoassay and MARKIT. Chemotherapy 30, 509-513 (1982)

13 Wright, P.J. and Plummer, D.J.: The use of urinary enzyme measurements to detect renal damage caused by nephrotoxic compounds. Biochem. Pharmacol. 23, 65-73 (1974)

14 Dance, N., Price, R.G., Robinson, D. and Stirling, J.L.: $\beta$-Galactosidase, $\beta$-glucosidase and $\mathrm{N}$-acetyl- $\beta$-glucosaminidase in human kidney. Clin. Chim. Acta 24, 189-197 (1969)

15 Kojima, R. and Suzuki, Y.: Studies on the nephrotoxicity of aminoglycoside antibiotics and protection from these effects (1). Nephrotoxicity of gentamicin and mercuric chloride. Folia Pharmocol. Japon. 84, 453-462 (1984) (Abs. in English)

16 Dellinger, P.. Murphy, T., Barza, M., Pinn, V. and Weinstein, L.: Protective effect of cephalothin against gentamicin-induced nephrotoxicity in rats. Antimicrob. Agents Chemother. 9, 172-178 (1976)

17 Dellinger, P., Murphy, T., Barza, M., Pinn, V. and Weinstein, L.: Effect of cephalothin on renal cortical concentrations of gentamicin in rats. Antimicrob. Agents Chemother. 9, 587-588 (1976)

18 Just, M. and Habermann, E.: The renal handling of polybasic drugs. 2 . In vitro studies with brush border and lysosomal preparations. Naunyn Schmiedebergs Arch. Pharmacol. 300, 67-76 (1977)

19 Silverblatt, F.J. and Kuehn, G.: Autoradiography of gentamicin uptake by the rat proximal tubule cell. Kidney Int. 5, 335-345 (1979)

20 Sastrasinh, M., Knauss, T., Weinberg, J.M. and Humes, H.D.: Identification of the aminoglycoside binding site in rat renal brush border membranes. J. Pharmacol. Exp. Ther. 222, 350358 (1982)

21 Luft, F.C. and Kleit, S.: Renal parenchymal accumulation of aminoglycoside antibiotics in rats. J. Infect. Dis. 130, 656-659 (1974)

22 Bennet, W.M., Hartnett, M.N., Gilbert, D., Houghton, D. and Porter, G.A.: Effect of sodium intake on gentamicin nephrotoxicity in the rat. Proc. Soc. Exp. Biol. Med. 151, 736-738 (1976)

23 Yoshida, T., Kimura, Y. and Tochino, T.: Pharmacokinetics of 6059-S in experimental animals. Chemotherapy 28 (s-7), 194-206 (1980)

24 Kaloyanides. G.J. and Pastoriza-Munoz, E.: Aminoglycoside nephrotoxicity. Kidney Int. 18, 571-582 (1980)

25 Giuliano, R.A., Paulus, G.J., Verpooten, G.A., Pattyn, V.M., Pollet, D.E., Nouwen, E.F., Laurent, G., Carlier, M.B., Maldague, P., Tulkens, P.M. and De Broe, M.E.: Recovery of cortical phospholipidosis and necrosis after acute gentamicin loading in rats. Kidney int. 26, 838-847 (1984)

26 Ngaha, E.O.: Further studies on the in vivo effect of cephaloridine on the stability of rat kidney Iysosomes. Biochem. Pharmacol. 31, 18431847 (1982)

27 Fry, M. and Plummer, D.T.: The stabilization of renal Iysosomes by cephaloridine: The role of a membrane-bound phospholipase $A_{2}$. In Nephrotoxicity. Edited by Fillastre, J.P.. p. 193-211. Masson Publishing U.S.A. Inc., New York (1978) 